\title{
Precursor B-Cell Lymphoblastic Primary Cardiac Lymphoma - Case Report and Review of Literature
}

Jayadev Manikkam Umakanthan ${ }^{1}$, Dipesh Uprety ${ }^{1 *}$, Keerthi Banda ${ }^{1}$, Navin Victor $^{1}$ and Herbert Auerbach ${ }^{2}$

${ }^{1}$ Department of Internal Medicine, Abington Memorial Hospital, 1200 Old York Road, Abington PA 19001, USA

${ }^{2}$ Department of Pathology, Abington Memorial Hospital, 1200 Old York Road, Abington PA 19001, USA

"Corresponding author: Dipesh Uprety, Department of Internal Medicine, Abington Memorial Hospital, 1200 Old York Road, Abington PA 19001, USA, Tel: 215-481-2000; E-mail: upretydipesh@gmail.com

Rec date: November 23, 2014, Acc date: January 22 2015, Pub date: January 31, 2015

Copyright: (c) 2015 Umakanthan JM, et al. This is an open-access article distributed under the terms of the Creative Commons Attribution License, which permits unrestricted use, distribution, and reproduction in any medium, provided the original author and source are credited.

\begin{abstract}
Primary cardiac lymphoma $(P C L)$ is an extremely rare malignancy and an uncommon presentation of NonHodgkin's Lymphoma. We report the first case of Precursor B-cell lymphoblastic PCL. A 44 year old Caucasian woman presented to our institution with one week history of worsening dyspnea, leg swelling and a weight gain of 12 pounds. Urgent echocardiography revealed pericardial effusion with tamponade physiology. Subsequent imaging showed a mass in the right atrio-ventricular groove. Pericardiocentesis was performed and cytopathology showed malignant appearing immature lymphocytes. Immunophenotypic analysis revealed $85 \%$ of the cells to be CD 19 and CD10 positive. The cells were negative for CD20, surface light chains, CD3 and CD5 consistent with immature Bcells. Terminal deoxynucleotidyl Transferase (TDT) was strongly positive confirming Precursor B-cell Lymphoblastic Lymphoma. Clinical presentation of PCL can be varied and can mimic non-neoplastic primary cardiac problems. When PCL is suspected and a pericardial effusion is present, pericardial fluid analysis can be a reasonable first step. If diagnostic, it can avoid further invasive procedures. Chemotherapy remains the standard treatment and should be initiated early given the aggressive nature of these high grade lymphomas. Surgery is needed only in selected cases with obstructive features.
\end{abstract}

Keywords: Primary cardiac lymphoma; Cardiac mass; Precursor BCell lymphoma; Pericardial effusion

\section{Introduction}

Primary cardiac lymphoma (PCL) is defined as a non-Hodgkin's lymphoma that involves only the heart and or pericardium or with an intra-pericardial location of the main tumor mass. PCL is a rare entity and majority is of the common histological type of Diffuse Large BCell Lymphoma (DLBCL) [1]. This article reports an extremely rare case of Precursor B-Cell Lymphoblastic Lymphoma presenting as PCL.

\section{Case Report}

A 44-year-old Caucasian woman with no significant past medical history presented to our emergency room with one week history of shortness of breath, bilateral leg swelling and a weight gain of 12 pounds. Her vital signs were stable. Physical examination revealed jugular venous distension, abdominal ascites and bilateral pedal edema. Her initial laboratory studies revealed a normal complete blood count, renal function and electrolytes and mildly elevated transaminases (AST $51 \mathrm{IU} / \mathrm{ml}$, ALT $94 \mathrm{IU} / \mathrm{ml}$ ). Her EKG showed normal sinus rhythm but with low voltage QRS Complexes. Chest Xray showed mild cardiomegaly. An urgent transthoracic echocardiogram revealed a large circumferential pericardial effusion with tamponade physiology. She underwent urgent pericardiocentesis with removal of $600 \mathrm{cc}$ of pericardial fluid. Initial cytology revealed a cell count of 36,000 WBC with 93\% blasts. Further testing included HIV, acute hepatitis Panel, HTLV I/II, Coxsackie virus A and B Ab,
ANA, ANCA, anti-DNA antibody, anti-CCP and Rheumatoid Factor. These were all negative. Serum Protein and Immuno electrophoreses did not show any abnormal immunoglobulins. Serum LDH was normal.

She continued to be dyspneic and a repeat echocardiogram showed moderate re-accumulation of the effusion but now revealing a homogenous mass along the anterior aspect of the right atrium. A cardiac MRI was performed which showed a mild enhancing mass in the right atrio-ventricular groove, measuring $2.2 \times 2.3 \mathrm{~cm}$ surrounding the right coronary artery from its origin (Figure 1).

Cytopathological evaluation of the pericardial fluid revealed a population of immature, intermediate to large sized lymphocytes with fine chromatin and small nucleoli (Figure 2). The cells lacked cytological features of a primary effusion lymphoma. Flow Cytometry revealed $85 \%$ of the lymphocytes to be CD19 and CD10 positive. CD20, surface light chains, CD3 and CD5 were negative. Terminal deoxynucleotidyl transferase (TdT) was positive (Figure 3). Immunophenotypically, the cells were consistent with a Precursor Bcell Lymphoblastic Lymphoma. And, Fluorescence in situ hybridization (FISH) Analysis for $\mathrm{t}(8,14)$ MYC Oncogene translocation was positive. However, the conventional cytogenetics was not performed.

Staging Computed Tomography scan of the neck, chest, abdomen and pelvis did not reveal any pathologically enlarged lymph nodes. A bone marrow biopsy was normal without evidence of lymphoma involvement. 
Citation: Umakanthan JM, Uprety D, Banda K, Victor N, Auerbach H (2015) Precursor B-Cell Lymphoblastic Primary Cardiac Lymphoma - Case Report and Review of Literature. J Blood Lymph 5: 133. doi:10.4172/2165-7831.1000133

Page 2 of 3

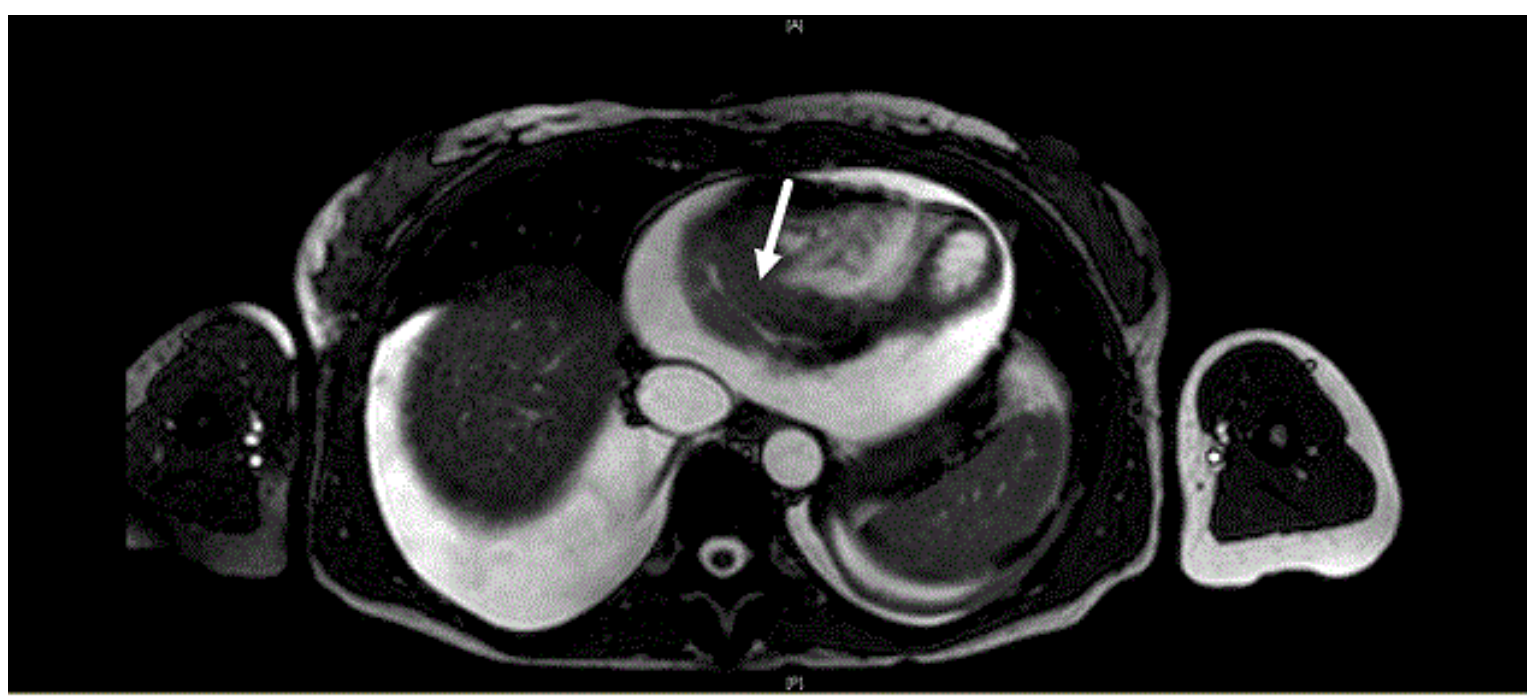

Figure 1: Cardiac Magnetic Resonance Imaging showing a mild enhancing mass in the right atrio-ventricular groove.

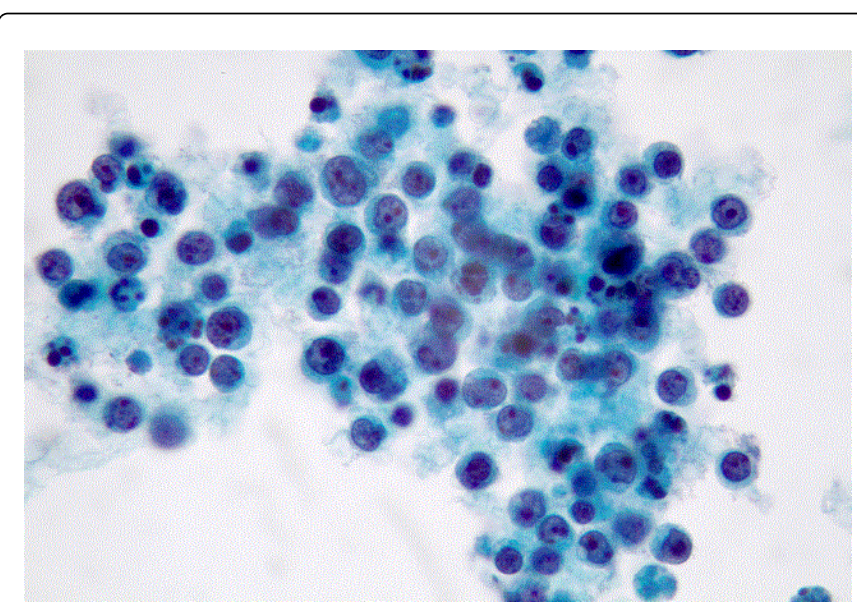

Figure 2: Pericardial fluid cytology showing a population of immature, intermediate to large sized lymphocytes with fine chromatin and small nucleoli.

Given the histology established in the pericardial effusion and the alarming location of the mass, a need for tissue biopsy was not indicated. Hence, a final diagnosis of Primary Cardiac Lymphoma of Precursor B-Cell Lymphoblastic type was made.

Patient was transferred to a tertiary center. A repeat echocardiogram showed modest re-accumulation and also rapid growth of the intra-cardiac mass now measuring $7 \times 2.3 \mathrm{~cm}$, invading the Right Atrial wall and compressing the Right Ventricle. She was immediately started on aggressive chemotherapy with Hyper CVAD (fractionated cyclophosphamide, vincristine, doxorubicin, dexamethasone) regimen with intrathecal prophylaxis. A repeat pericardiocentesis and fluid Flow Cytometry showed variable weak CD20 expression and hence Rituximab was added. After 4 weeks, reimaging with a cardiac MRI showed complete resolution of the mass and the pericardial effusion. She is alive at 5 months from diagnosis currently undergoing chemotherapy with the intention of allogeneic hematopoietic cell transplantation.

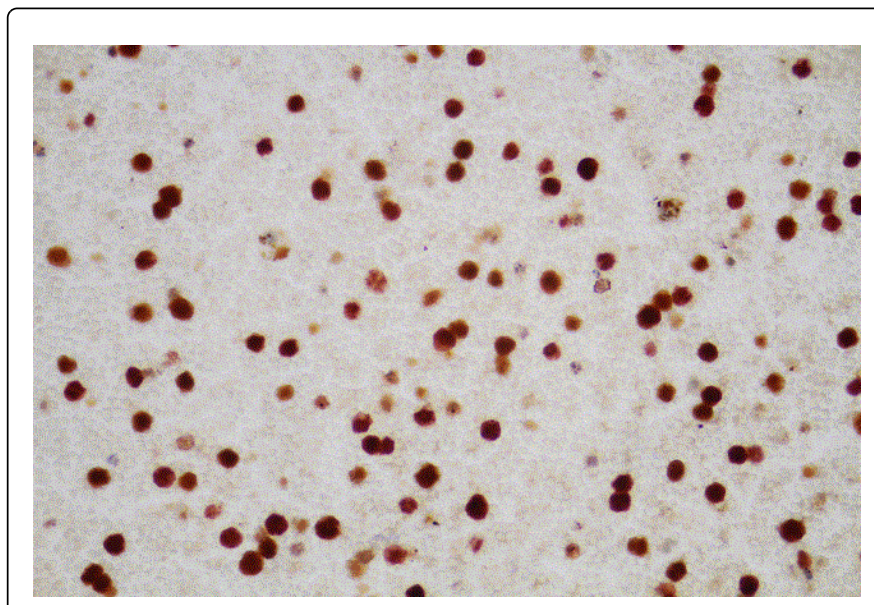

Figure 3: Photomicrograph showing Terminal deoxynucleotidyl transferase (TdT) positivity.

\section{Discussion}

PCL is a very rare malignancy and an uncommon presentation of lymphomas. The largest review of literature on PCL so far is by [1] reporting 197 cases followed by [2] with 56 cases (these cases also included in the former review)[1] found that majority were DLBCL compared to a very small proportion of Burkitt's, T - Cell, Small lymphocytic and Plasmablastic lymphoma. In [2] review, similar findings had been reported with majority of DLBCL and only a few rare variants of non-cleaved small cells and immunoblastic lymphomas. Few reports of T-Cell Lymphomas have been reported $[3,4]$. Otherwise, to the best of our knowledge, Precursor B-cell Lymphoblastic PCL has not been reported in the English literature. 
Clinical presentation of PCL can be varied and can mimic nonneoplastic primary cardiac problems. Reports of patients presenting with heart failure, cardiac tamponade, SVC Syndrome, arrhythmias and other conduction abnormalities or even sudden cardiac arrest have been documented. In our case, the mass was non-obstructing and did not cause any electrical conduction abnormalities. However, the significant pericardial effusion caused tamponade leading to a presentation of rapidly progressing heart failure.

Obtaining histopathological specimen early is crucial for management. In the presence of pericardial effusion, diagnostic pericardiocentesis is a reasonable first step. In our case, the tissue sample was not obtained as the pericardial fluid confirmed the diagnosis. Hence, it avoided further invasive procedures with potential complications especially given the location of the tumor. However, one third of effusion fluid cytology can be non-diagnostic $[5,6]$. In such cases or in the absence of an effusion, tissue biopsy by transvenous intracardiac biopsy using fluoroscopic and/or ultrasound-guidance can be performed [6]. We did not perform the conventional cytogenetics study on the blasts from the pericardial fluid. This is one of the limitations of our study.

In our case, positive FISH Analysis for $\mathrm{t}(8,14)$ MYC Oncogene raised the possibility of Burkitt's Lymphoma (BL). However, there have been studies showing aberrant erroneous translocations and juxtaposition of the MYC in a small fraction of adults with B-cell LBL [7]. On the other hand, studies have also shown aberrant immunophenotypes in BL as well [8]. But, in this case there was unequivocal strong expression of TDT which is almost universally expressed in Acute Lymphoblastic Lymphoma and is reportedly lacking in BL. This is the only reliable criterion in the differential diagnosis [8]. Also, the absence of CD20 and surface light chain expression combined with the positive TdT expression further supports the diagnosis of Precursor B-cell Lymphoblastic Lymphoma with a $t(8,14)$ MYC Oncogene translocation as opposed to the converse (Burkitt's with an immature immunophenotype).

PCL can be very aggressive and should be considered an oncologic emergency [9]. In our patient, the mass had doubled in size within a span of one week reiterating this fact. Primary treatment modality is Chemotherapy. Surgical intervention with tumor debulking is needed only in cases of large tumors causing mechanical obstruction [10-13]. Radiotherapy has been given in selected cases. Overall, there is no evidence that PCL should be treated differently from other bulky aggressive lymphomas arising at other anatomic sites [5]. Of all the modalities used in the past, Chemotherapy seems to have the greatest effect on survival [1]. The choice of chemotherapy depends on the histological subtype. As vast majority are diffuse large B-Cell Lymphomas (Strongly CD 20+), Rituximab based R-CHOP (rituximab, cyclophosphamide, doxorubicin, vincristine, prednisolone) is the most widely used regimen. Our patient was started on Hyper-CVAD regimen due to the Precursor B-Cell Lymphoblastic histology.

\section{Conclusion}

PCL is a rare malignancy and also a rare presentation of a lymphoma. Clinical presentations can be varied and can mimic cardiac problems. Hence, a high index of suspicion is warranted for the early diagnosis. When PCL is suspected and a pericardial effusion is present, Pericardiocentesis and fluid analysis can be a reasonable first step, avoiding further invasive procedures. Chemotherapy continues to be the primary treatment modality and should be initiated early given the aggressive nature of these high grade lymphomas.

\section{Funding}

Department of Internal Medicine, Abington Memorial Hospital, Abington, PA, USA.

\section{References}

1. Petrich A, Cho SI, Billett H (2011) Primary cardiac lymphoma: an analysis of presentation, treatment, and outcome patterns. Cancer 117: 581-589.

2. Anghel G, Zoli V, Petti N, Remotti D, Feccia M, et al. (2004) Primary cardiac lymphoma: report of two cases occurring in immunocompetent subjects. Leuk Lymphoma 45: 781-788.

3. Nascimento AF, Winters GL, Pinkus GS (2007) Primary cardiac lymphoma: clinical, histologic, immunophenotypic, and genotypic features of 5 cases of a rare disorder. Am J Surg Pathol 31: 1344-1350.

4. Giunta R, Cravero RG, Granata G, Sellitto A, Romano C, et al. (2004) Primary cardiac T-cell lymphoma. Ann Hematol 83: 450-454.

5. Ceresoli GL, Ferreri AJ, Bucci E, Ripa C, Ponzoni M, et al. (1997) Primary cardiac lymphoma in immunocompetent patients: diagnostic and therapeutic management. Cancer 80: 1497-1506.

6. Faganello G, Belham M, Thaman R, Blundell J, Eller T, et al. (2007) A case of primary cardiac lymphoma: analysis of the role of echocardiography in early diagnosis. Echocardiography 24: 889-892.

7. Burmeister T, Molkentin M, Schwartz S, Gökbuget N, Hoelzer D, et al. (2013) Erroneous class switching and false VDJ recombination: molecular dissection of $\mathrm{t}(8 ; 14) / \mathrm{MYC}-\mathrm{IGH}$ translocations in Burkitt-type lymphoblastic leukemia/B-cell lymphoma. Mol Oncol 7: 850-858.

8. Kelemen K, Braziel RM, Gatter K, Bakke TC, Olson S, et al. (2010) Immunophenotypic variations of Burkitt lymphoma. Am J Clin Pathol 134: $127-138$.

9. Stein M, Zyssman I, Kantor A, Spencer D, Lewis D, et al. (1994) Malignant lymphoma with primary cardiac manifestations: a case report. Med Pediatr Oncol 22: 292-295.

10. Kawamura T, Sakaguchi T, Nishi H, Miyagawa S, Yoshikawa Y, et al. (2013) Successful treatment of a large primary cardiac lymphoma by surgical resection combined with chemotherapy: report of a case. Surg Today 43: 1066-1070.

11. Yanase Y, Yamauchi A, Uehara M, Tachibana K, Muraki S, et al. (2012) Surgical resection of primary cardiac lymphoma presenting as a huge mass: report of two cases. Gen Thorac Cardiovasc Surg 60: 494-497.

12. Chiu KM, Lin TY, Li SJ, Chan CY, Chu SH (2006) Surgical resection of primary cardiac lymphoma. Clin Lymphoma Myeloma 6: 420-421.

13. Gosev I, Siri F, Gasparovi H, Ugljen R, Nola M, et al. (2006) Surgical treatment of a primary cardiac lymphoma presenting with tamponade physiology. J Card Surg 21: 414-416. 